\title{
Extremum Seeking Control for Truck Drag Reduction
}

\author{
Georgios Papageorgiou, Jason Barden, James F. Whidborne, Kevin P. Garry \\ School of Aerospace, Transport and Manufacturing \\ Cranfield University \\ Bedfordshire, United Kingdom \\ j.f.whidborne@cranfield.ac.uk
}

\begin{abstract}
The aerodynamic drag on a heavy truck tractor and semi-trailer combination can be reduced by means of a wind deflector installed on the roof of the tractor cab. The drag reduction is dependent upon the height and shape of the deflector. A variable height deflector has been constructed and tested in a wind-tunnel and on-road. In this paper, an extremumseeking control scheme is proposed to adjust on-line the deflector height to minimize the aerodynamic drag. The effectiveness of the scheme is evaluated by simulation and its practicality is evaluated.
\end{abstract}

Index Terms-drag reduction, flow control, extremum seeking

\section{INTRODUCTION}

The geometry of typical European Heavy Goods Vehicle (HGV) tractor-trailer combinations is primarily driven by operational needs. This means that they are aerodynamically inefficient due to their bluff geometry. Consequently vehicle manufacturers and aftermarket companies produce a variety of add-on aerodynamic devices in an effort to reduce the aerodynamic drag.

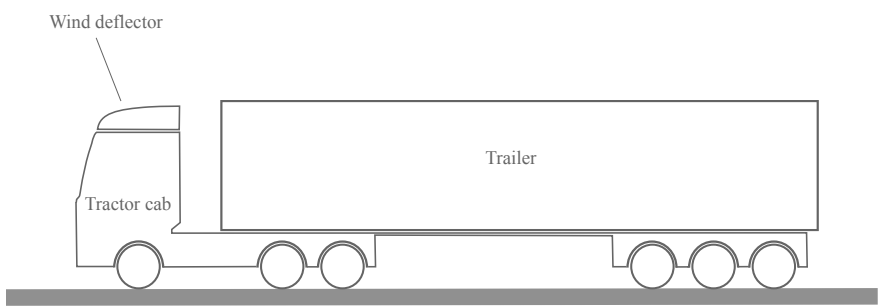

Fig. 1. Schematic of tractor-trailer combination with cab mounted roo deflector.

Probably the most common and efficient add-on device is the cab mounted roof deflector, as shown in Figure 1. It has been shown in wind tunnel experiments that it can contribute between 20 and $30 \%$ drag reduction over a baseline model [1], [2]. The use of air deflectors on modern HGVs can be attributed to Saunders [3].

Another early study is by Buckley et al [4]. A history [5] of efforts in drag reduction for commercial vehicles includes details of other studies. Other early cab mounted designs include Beers [6] and Lissaman \& Lambie [7]. Variations in

This work was supported in part by Hatcher Components Limited, Mercedes Benz UK Ltd and the Technology Strategy Board under TSB Contract: $\mathrm{TP} / 9 / \mathrm{PCV} / 6 / 150059 \mathrm{~B}$ these designs were tested by Hucho [8] whose results show that the drag reduction depends heavily on the deflector design. The efficiency of the deflector varies by as much as $19 \%$ solely due to the geometry of the deflector.

A particular deflector geometry is usually designed for a specific tractor-trailer configuration. However commercial haulage companies often use different trailer units with various heights and shapes with the same tractor unit, which usually means a decrease in effectiveness. Furthermore the deflector efficiency is dependent upon the wind yaw angle. A vehicle experiences its minimum aerodynamic drag with zero wind yaw angle [9], and a change in the wind direction results in an increase in the aerodynamic drag and a possible change in the optimum deflector height [10]. In most applications the cab mounted roof deflectors have a fixed functional height, hence there is a potential for greater efficiency with a variable height deflector [1]. HGVs operate for extended periods of time facing a continuous variation in wind speed and direction. Thus, static air deflectors provide the maximum possible drag reduction only for a restricted range of wind direction and lose efficiency when significant wind yaw angle is present [11]. Hence there is a need for deflectors that can adapt their geometry in some way to suit the trailer geometry and the yaw angle. The most straightforward way to do this is by varying the deflector height [10]. Hence a cab-mounted, variable height deflector named 'FREDDIE' (Fuel REDucing DevIcE) has been designed and road-tested [10]. The deflector height is scheduled based on the wind yaw angle obtained from pressure measurements. The schedule is determined from wind tunnel experiments and has been validated with road tests [10]. However it is an open loop control scheme.

Drag minimization schemes can be provided through feedback control. For example, Seifert et al. [12] demonstrated net drag reduction of $10 \%$ using blowing and suction devices attached to the rear of the trailer. In particular, Extremum Seeking Control (ESC) has been proposed to adapt the control to minimize the drag in bluff bodies. Henning et al. [13] combined a model-based sensor and an ESC extended with Kalman filter, to determine the optimal phase of an actuation to synchronise the vortex shedding for drag reduction of a bluff body with a blunt trailing edge in wind tunnel experiments. Beaudoin et al. [14] investigated the ESC method proposed by Ariyur and Krstic [15] to control the drag of a bluff geometry 
using strain gauge measurements and a rotating cylinder actuator located on the rear edge. Experiments were conducted that demonstrated the scheme. Henning et al. [16] also used an extended Kalman filter and found it to be faster than the estimation of the standard algorithm in two-dimensional bluff body and a generic three-dimensional car model experiments. Additionally, Beaudoin et al. [17] conducted experiments in order to record the relation of drag reduction of a bluff body with the angle of vortex generators mounted on the bluff body. The utilization of the ESC method contributed to the fast prediction of the optimal vortex generators angle and the fast transition of the angle in different freestream velocities. Pastoor et al. [18] used pressure gauge measurements along with the ESC scheme proposed by Ariyur and Krstic [15] to minimize drag reduction of a bluff body. The scheme was tested in a wind tunnel with good results. Another experimental study [19] used ESC to reduce both lift and drag for a bluff body. Drag and lift were not explicitly measured, but hot wire anemometry was used to detect separation. Brackston et al. [20] examined experimentally their modified extremum seeking method to an open-loop control system, an axisymmetric bluff-body wake, forced by a pulsed jet and showed that the control scheme is able to adapt to changing conditions.

Although ESC has been used for bluff body drag reduction, previously is does not seem to have been applied for the HGV problem, in particular for adaptive control of cab mounted deflectors. In this work, the suitability of an ESC method that continuously varies the height of a cab mounted roof deflector so that the minimum drag is achieved despite variations in the wind yaw angle and uncertainty regarding the geometry of the trailer is tested by means of simulation. The ESC method is due to Ariyur and Krstic [15]. The model is based on wind tunnel measurements of a scale model of a tractor-trailer combination with the 'FREDDIE' variable height deflector described earlier [10]. It is assumed that a measurement of the wind drag is available. In the next section, the ESC scheme is summarised. In Section III, the experiments to obtain the aerodynamic characteristics of the deflector and tractor-trailer combination that are used for the simulations are summarized. The simulation model is described in Section IV and some results are presented in Section V. Finally the proposed scheme is discussed and limitations and implementation problems identified.

\section{Extremum SEEKING CONTROL}

In many engineering problems, it important that the system operates at an optimal condition. However this optimal operating condition or point is sometimes not known a priori. For such problems, the extremum seeking provides a closed-loop control method that can be used to find the optimal operating point on-line whilst maintaining stability and boundedness of the signals [21]. Furthermore, the method can find the optimal point by simply using the output measurements of the system plant.

Consider the SISO nonlinear plant model

$$
\dot{x}=f(x, u), \quad y=h(x),
$$

where $x \in \mathbb{R}^{n}$ is the state, $u \in \mathbb{R}$ is the control input, $y \in \mathbb{R}$ is the measured output. Furthermore, $f: \mathbb{R}^{n} \times \mathbb{R} \rightarrow \mathbb{R}^{n}$ and $h: \mathbb{R}^{n} \rightarrow \mathbb{R}$ are assumed smooth. The smooth state-feedback control law

$$
u=\alpha(x, \theta)
$$

is assumed to stabilize the system for any equilibrium that the parameter $\theta$ can produce.

The scheme used here is the perturbation-based gradient ESC proposed by Ariyur and Krstic [15] and is shown in Figure 2. It is an extension of a simple method for seeking extrema of static nonlinear maps [22]. A proof of the stability of the scheme is given in [23]. It is possibly the most applied and studied among all the extremum seeking schemes; its major advantages are the fast adaptation and the simple implementation [21].

In order to explain how the approach works, let us simplify the approach by assuming that the nonlinear plant given by (1) consists of just a static, constant, non-linear map $y=f(\theta)$. The explanation follows that of [15] where a detailed and rigorous analysis can be found. The purpose of the algorithm is to minimise the difference $\theta-\theta^{*}$, where $\theta^{*}$ is the optimal input, that being the input that drives the output $y$ to a minimum. The scheme uses a sinusoidal perturbation signal, $a \sin (\omega t)$, to generate an estimate of the gradient information of the static cost function, $f(\theta)$.

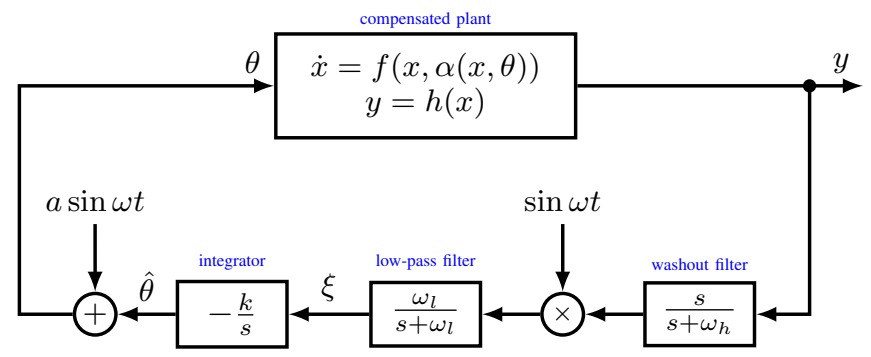

Fig. 2. Gradient extremum seeking control scheme.

Given a static map function $f(\theta)$, performing a Taylor Series expansion about a minimum at $\theta=\theta^{*}$, neglecting the third order and higher terms and noting that $\theta^{*}$ is a minimum hence $f^{\prime}(\theta)=0$ gives

$$
f(\theta)=f^{*}+\frac{1}{2} f^{\prime \prime}\left(\theta-\theta^{*}\right)^{2} .
$$

Let $\hat{\theta}$ denote the estimate of the unknown optimal input $\theta^{*}$ and let $\tilde{\theta}$ denote the estimation error, that is $\tilde{\theta}=\theta^{*}-\hat{\theta}$. From Figure 2, $\theta=a \sin (\omega t)+\hat{\theta}$. Expanding gives $\theta-\theta^{*}=$ $a \sin (\omega t)-\tilde{\theta}$ and substituting into (3) gives

$$
y=f^{*}+\frac{1}{2} f^{\prime \prime}(\tilde{\theta}-a \sin \omega t)^{2} .
$$

Using the trigonometric identity $2 \sin ^{2}(\omega t)=1-\cos (2 \omega t)$ gives

$y=f^{*}+\frac{1}{4} a^{2} f^{\prime \prime}+\frac{1}{2} f^{\prime \prime} \tilde{\theta}^{2}-a f^{\prime \prime} \tilde{\theta} \sin (\omega t)+\frac{1}{2} a^{2} f^{\prime \prime} \cos (2 \omega t)$.

The measured output $y$ then is passed through a washout filter $s /\left(s+\omega_{h}\right)$ which removes the steady state component, 
$f^{*}+a^{2} f^{\prime \prime} / 4$, which does not carry any information about the gradient [24]. Subsequently the signal is multiplied with a unit sinusoidal signal, $M(t)=\sin (\omega t)$, and passed through a low pass filter $\omega_{\ell} /\left(s+\omega_{\ell}\right)$. After applying the trigonometric identities, $2 \sin ^{2}(\omega t)=1-\cos (2 \omega t)$ and $2 \cos (2 \omega t) \sin (\omega t)=$ $\sin (3 \omega t)-\sin (\omega t)$, the output of the low-pass filter is approximately given by

$$
\begin{array}{r}
\xi=-\frac{1}{2} a f^{\prime \prime} \tilde{\theta}+\frac{1}{2} a f^{\prime \prime} \tilde{\theta} \cos (2 \omega t)+\frac{1}{8} a^{2} f^{\prime \prime}(\sin (\omega t)-\sin (3 \omega t) \\
+\frac{1}{2} f^{\prime \prime} \tilde{\theta}^{2} \sin (\omega t) .
\end{array}
$$

The signal is passed through a negative gain integrator, this averages out the sinusoidal signals, the result being dominated by the steady state signals, thus

$$
\hat{\theta}=\frac{1}{2} k \int a f^{\prime \prime} \tilde{\theta} \mathrm{d} t
$$

or $\dot{\hat{\theta}}=k a f^{\prime \prime} \tilde{\theta} / 2$. Now we have assumed that $f(\theta)$ is a constant map so $\theta^{*}$ is constant, and since $\tilde{\theta}=\theta^{*}-\hat{\theta}$, then $\dot{\tilde{\theta}}=-\dot{\hat{\theta}}$ giving $\dot{\tilde{\theta}}=-k a f^{\prime \prime} \tilde{\theta} / 2$. Since $k>0, a>0$ and $f^{\prime \prime}>0, \tilde{\theta}$ will converge asymptotically to zero and $\hat{\theta}$ converges to $\theta^{*}$.

The design parameters of the control scheme are user defined but some general rules have to be met. The perturbation frequency $\omega$ has to be sufficiently greater that the washout filter frequency $\omega_{h}$, so that the filter eliminates the DC component in $y$ without corrupting the estimation of the gradient $f^{\prime}(\theta)$ [24]. An increased $\omega$ permits faster adaptation but a large value may cause instability. An inadequate choice of $a$ results in a slow convergence rate and there is the danger for the solution to be stuck in a local minimum. A larger value of $a$ increases the convergence rate but simultaneously is accompanied by greater oscillations. The integrator gain $k$ affects radically the convergence rate but a high value of $k$ may cause disturbances and instability

\section{AERodynAmic Model ExPERIMENTS}

A 1/8th scale model of a Mercedes-Benz Actros commercial vehicle made from high density modeling foam was used in the experimental test. The vehicle was fitted with a cab-mounted adjustable deflector model of 'FREDDIE' made from vacuumformed, high impact, polystyrene sheet. The deflector could vary in height from $484 \mathrm{~mm}$ to $514 \mathrm{~mm}$ measured from the ground to the top of the deflector. This corresponded to a full-scale height range of $3.9 \mathrm{~m}$ to $4.1 \mathrm{~m}$. The deflector was adjusted using $3 \mathrm{~mm}$ medium-density fibreboard spacers. The number of the spacers was 10 and the maximum extension of the deflector was $30 \mathrm{~mm}$ or $24 \mathrm{~cm}$ in full-scale dimensions.

The model was tested in the Cranfield $8^{\prime} \times 6^{\prime}$ low speed wind tunnel. The maximum wind tunnel speed is $55 \mathrm{~ms}^{-1}$ and the closed working section is $2.4 \mathrm{~m} \times 1.8 \mathrm{~m}$. Boundary layer suction is fitted to minimise the effects of the boundary layer growth on the ground board when used for force measurements. Figure 3 shows the model in the wind tunnel working section. For the drag force measurements, the scale model was placed on a rotating ground board with force-torque sensors. The rotation yaw angle range is $-17.5^{\circ} \leq \beta \leq 17.5^{\circ}$.

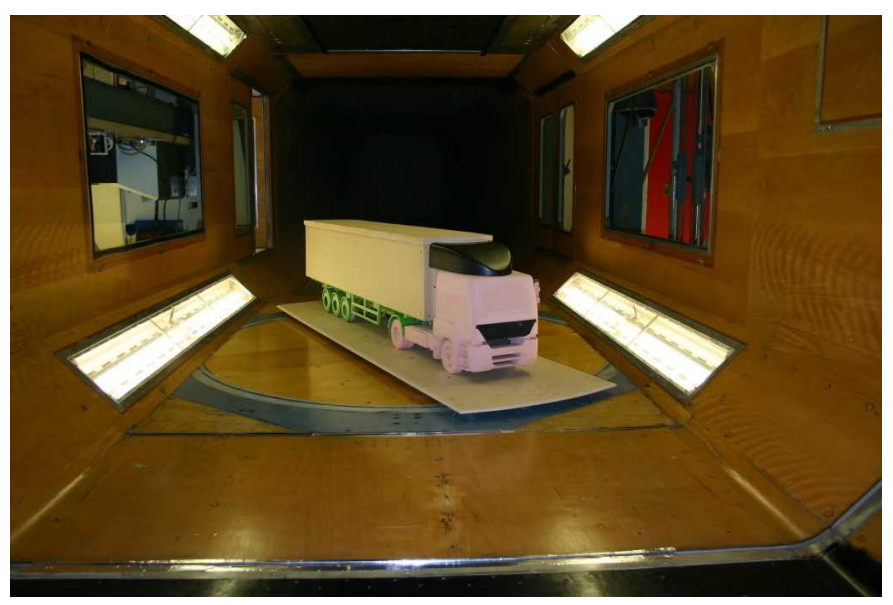

Fig. 3. Cranfield 8' x 6' model installation arrangement.

Six component force-torque sensors were used to measure the force and moment loads for all axes. Data for various wind yaw angles were obtained for several tractor to trailer separation ratios. Experimental details and the data can be found in [10].

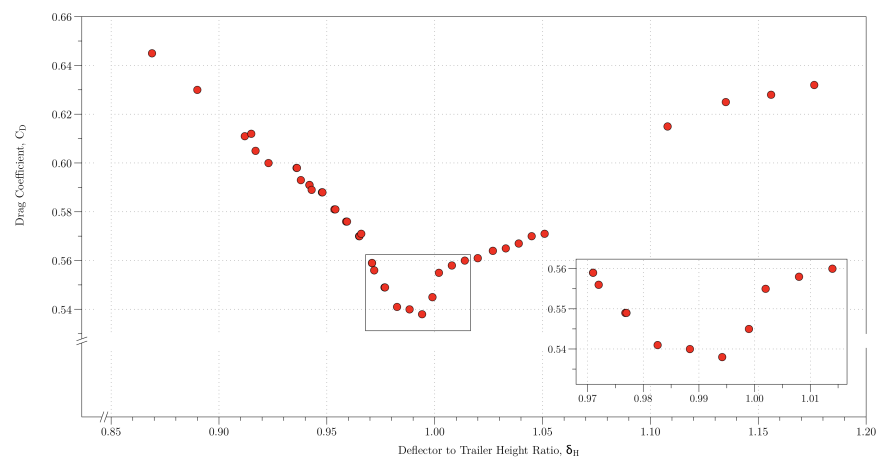

Fig. 4. Drag coefficient, $C_{D}$, variation with deflector height ratio, $\delta_{H}$, for zero yaw.

The variation of drag coefficient, $C_{D}$, with the deflector height ratio, $\delta_{H}$, is shown in Figure 4 for the yaw angle fixed at $0^{\circ}[10$, p. 140]. The deflector height ratio is defined by $\delta_{H}=d_{1} / d_{2}$ where $d_{1}$ is half the deflector height and $d_{2}$ is half the trailer height as shown in Figure 5. The tractor to trailer separation ratio is defined by $\delta_{g}=d_{g} / d_{2}$ where $d_{g}$ is the tractor to trailer separation distance shown in Figure 5, and was fixed at $345 \mathrm{~mm}$, giving $\delta_{g}=1.335$.

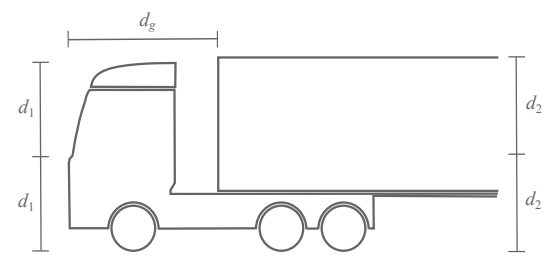

Fig. 5. Model dimensions.

The results indicated that for a given trailer height and separation, at $0^{\circ}$ yaw angle, there exists an optimum deflector height that produces the lowest drag configuration. The deflec- 
tor height ratio $\left(\delta_{H}\right)$ tested that produced the lowest $C_{D}$ was 0.994 , approximately the same height as the trailer. The $C_{D}$ was very sensitive to $\delta_{H}$ either side of this optimum, a rise in $C_{D}$ of $18 \%$ was obtained for a $0.1(10 \%)$ change in $\delta_{H}$. This result highlights the importance of the optimum deflector height on the overall drag of the vehicle.

\section{Simulation Model}

For the simulation, the plant model given by (1) consists of a model of the surge dynamics of the heavy vehicle combined with drag characteristics represented by look-up tables of the data obtained from the wind tunnel along with a cruise control.

\section{A. Surge dynamics}

It is assumed that the surge dynamics are decoupled from motion modes, hence from Newton's second law of motion, the surge dynamics are given by

$$
m_{T} \frac{\mathrm{d} V_{T}}{\mathrm{~d} t}=f_{t}-\left(f_{D}+f_{M}\right)
$$

where $V_{T}$ is the truck surge velocity (forward truck speed), $m_{T}$ is the total mass of the truck, $f_{t}$ is the traction force, $f_{M}$ is the gravitational and rolling resistance force and $f_{D}$ is the aerodynamic drag force described in the next subsection.

The gravitational and rolling resistance force is given by

$$
f_{M}=m_{T} g\left(\sin \alpha+\left(C_{r r}+C_{r v} V_{T}^{2}\right) \cos \alpha\right)
$$

where $\alpha$ is the road gradient, $g$ is the gravitational constant, $C_{r r}$ is the rolling resistance coefficient and $C_{r v}$ is the additional velocity dependent rolling resistance coefficient that is mostly due to aerodynamic drag on the tyre [25].

The engine traction force, $f_{t}=k_{T} T$, is assumed to be dependent on a throttle setting $T \in[0,1]$ where $k_{T}$ is the throttle to engine force gain.

\section{B. Aerodynamic drag model}

The aerodynamic drag force is given by

$$
f_{D}=\frac{1}{2} \rho V_{R}^{2} A C_{D}\left(\delta_{H}, \beta\right)
$$

where $\rho$ is the air density, $V_{R}$ is the wind speed incident on the truck, $A$ is the truck reference area and $C_{D}$ is the drag coefficient that is dependent on the deflector height ratio, $\delta_{H}$, and the sideslip angle, $\beta$. The incident wind speed depends on the truck velocity, wind speed, $V_{W}$, and direction, $\phi$ relative to the truck velocity, and is given by

$$
V_{R}=\sqrt{V_{T}^{2}+V_{W}^{2}+2 V_{T} V_{W} \cos \phi} .
$$

The sideslip angle is given by $\beta=\arcsin \left(V_{W} / V_{R} \sin \phi\right)$.

The value $C_{D}$ is calculated using the Simulink Lookup Table (n-D) block with 2 dimensions with cubic spline interpolation and linear extrapolation. The look-up table data is generated using the fit surface fitting tool from the MATLAB Curve Fitting Toolbox using the data from [10] and using piecewise linear interpolation. Four additional points were introduced at the domain vertices to extend the curve to the full domain. Linear interpolation is used for the surface fitting

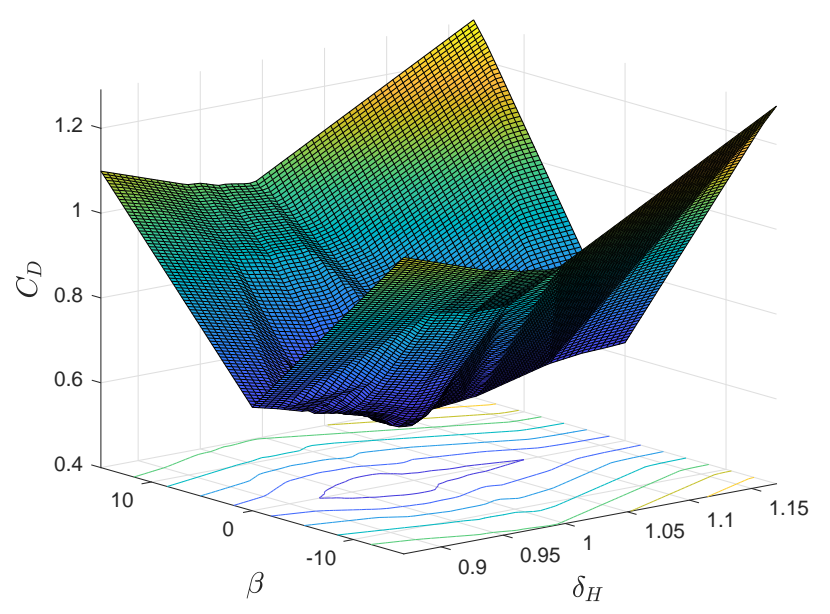

Fig. 6. Mapping $\left(\delta_{H}, \beta\right) \mapsto C_{D}$.

rather than higher order schemes to avoid the introduction of local minima. The surface fit mapping is shown in Figure 6.

It is assumed that the drag, $f_{D}$, can be directly measured. In the simulation, the measurement is in units of $\mathrm{kN}$. Generally the measurements would be subject to noise, however the results dispayed in the next section assume that there is no noise on the measurements.

\section{Speed control}

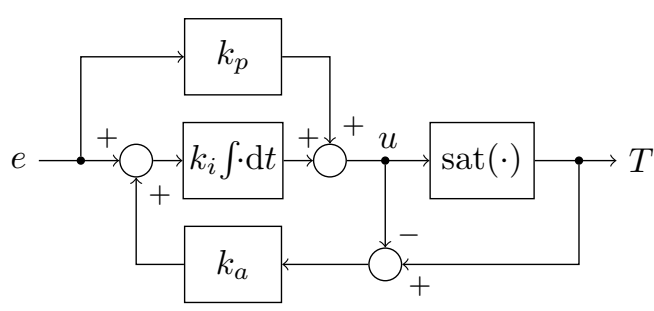

Fig. 7. PI speed controller with antiwindup.

The throttle setting, $T$, is set by a PI controller with antiwindup [26, pp 634-635] that represents either a cruise controller or the behaviour by a driver in maintaining a constant speed. The block diagram is shown in Figure 7, where $k_{p}$ and $k_{i}$ are the standard gain terms of a PI controller, $k_{a}$ is the antiwindup feedback gain, sat $(\cdot)$ is a saturation operator defined by

$$
\operatorname{sat}(x)=\left\{\begin{array}{l}
1 \text { if } x \geq 1 \\
0 \text { if } x \leq 0 \\
x \text { otherwise }
\end{array}\right.
$$

and $e(t)=V_{r}-V_{T}$ is the speed error with $V_{r}$ being the reference truck speed.

\section{ESC}

The feedback is completed by the ESC described in Section II with the ESC control output $\theta$ being the desired deflector height ratio, $\delta_{H}$. The aim is to minimize the aerodynamic drag by varying the deflector height. It is assumed that the 
aerodynamic drag force, $f_{D}$, can be directly measured and is the input to the ESC. Note that the ESC integrator also requires antiwindup in a similar manner to the speed controller.

\section{Simulation Results}

The truck model, ESC controller and speed controller are coded in Simulink. ${ }^{1}$ The constants used for the model are given in Table I. It is assumed that the measurements are noise-free.

TABLE I

SIMULINK SIMULATION COEFFICIENTS

\begin{tabular}{ll}
\hline Total mass, $m_{T}$ & $20 \times 10^{3} \mathrm{~kg}$ \\
Gravitational constant, $g$ & $9.81 \mathrm{~m} \mathrm{~s}^{-2}$ \\
Rolling resistance coefficient, $C_{r r}$ & $6.0 \times 10^{-3}$ \\
Velocity dependent rolling resistance coefficient, $C_{r v}$ & $10.0 \times 10^{-6}$ \\
Truck reference area, $A$ & $4.0 \mathrm{~m}^{2}$ \\
Air density, $\rho$ & $1.22 \mathrm{~kg} \mathrm{~m}^{-3}$ \\
Throttle to engine force gain, $k_{T}$ & $12 \times 10^{3} \mathrm{~N}$ \\
ESC perturbation amplitude, $a$ & 0.01 \\
ESC perturbation frequency, $\omega$ & $5.0 \mathrm{rad} \mathrm{s}$ \\
ESC integrator gain, $k$ & -5.0 \\
ESC antiwindup gain, $k_{w}$ & -5.0 \\
ESC high-pass filter coefficient, $\omega_{h}$ & 0.1 \\
ESC low-pass filter coefficient, $\omega_{\ell}$ & 0.5 \\
Speed controller proportional gain, $k_{p}$ & 1.5 \\
Speed controller integral gain, $k_{i}$ & 0.3 \\
Speed controller antiwindup gain, $k_{a}$ & 1.0 \\
ESC antiwindup maximum, $\theta_{\max }$ & 1.165 \\
ESC antiwindup minimum, $\theta_{\min }$ & 0.875 \\
\hline
\end{tabular}

\section{A. ESC controller convergence}

The first simulation case illustrates the convergence of the ESC routine. The speed controller reference, $V_{r}$, is set to $24 \mathrm{~ms}^{-1}$, but the initial value of the ESC controller integrator is set to the limits of travel of $\delta_{H}$, that being the lowest $\delta_{H}=0.88$ and highest $\delta_{H}=1.16$. The gradient and wind speed are set to zero. The resulting responses of the ESC controller output, $\theta$, which is the demand on $\delta_{H}$, is shown in Figure 8, along with the optimal value of $\delta_{H}$ for $\beta=0$, i.e. $\delta_{H}$ corresponding to minimal $C_{D}$, that is $\delta_{H}^{0}=0.9925$. The response shows convergence within $25 \mathrm{~s}$ with the final output being the expected oscillation about $\delta_{H}^{0}$.

\section{B. Performance with speed disturbance}

For the next case the performance of the scheme is for demanded vehicle speed changes. The test is again performed in still air and zero gradient. The speed demand and response are shown in Figure 9. Observe that the speed controller appears slew-rate limited, this is a result of the antiwindup scheme described in Section IV-C.

The deflector response is shown in Figure 10. The ESC controller output, $\theta=\delta_{H}$, maintains the tracking of the optimal value, $\delta_{H}^{0}$. However the changes in the drag result in perturbations on the magnitude of the control perturbation signal during the transients; with a decreasing magnitude for decreasing speed and vice versa. The perturbations decay about $25 \mathrm{~s}$ after the transient, which is the expected rate from the ESC controller convergence case.

\footnotetext{
${ }^{1}$ Data and MATLAB/Simulink simulation codes are available on doi:10.17862/cranfield.rd.6491846
}

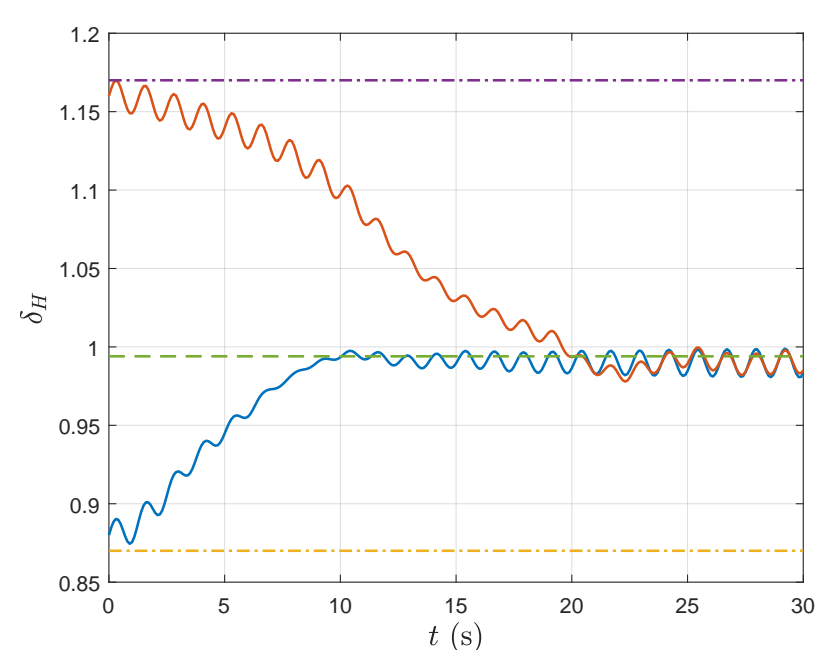

Fig. 8. ESC controller output, $\theta(-)$, converging to the optimal value $\delta_{H}^{0}$ $(---)$. The travel limits are also shown $(-\cdot-\cdot-)$.

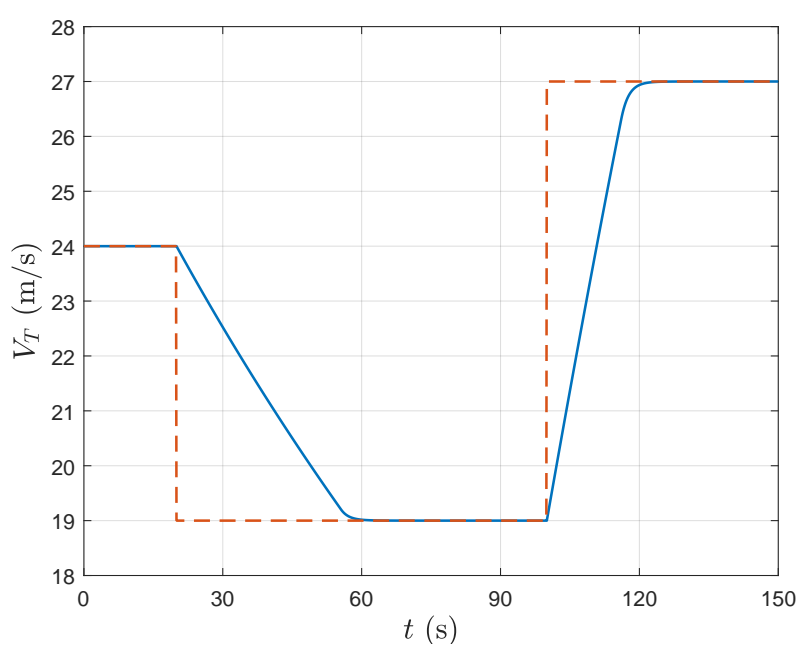

Fig. 9. Vehicle speed demand and response

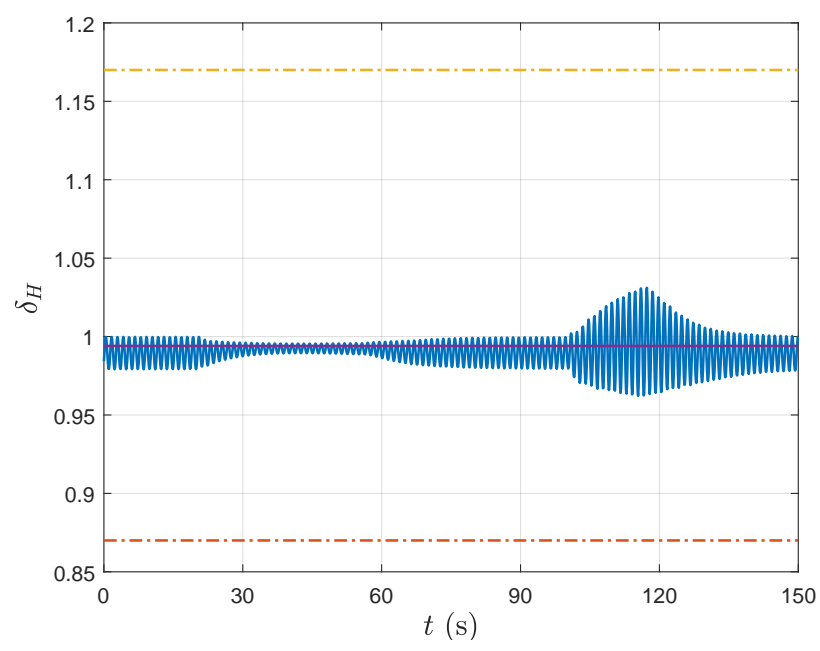

Fig. 10. ESC controller output, $\theta$, following the optimal value $\delta_{H}^{0}$. 


\section{CONCLUSIONS}

The simulations show that the scheme works in principle, however, only a limited set of simulations are shown. There are a number of limitations. The controller converges in about $25 \mathrm{~s}$, and this is probably too slow to be practical. Tests of a Newton-based ESC scheme due to [27] are presented in [28], and these increase the convergence rate, but not dramatically. It appears that the main constraint on the convergence rate is the ESC perturbation frequency, $\omega$. The convergence rate increases with the frequency, however, the maximum frequency is constrained by the maximum bandwidth of the actuating mechanism; frequencies higher than $5 \mathrm{rad} / \mathrm{s}$ are probably impractical. The second simulation case shows that the scheme is robust to speed changes. However, the scheme appears to be very sensitive to changes in the wind direction; a $45^{\circ}$ change in the wind yaw causes the ESC to destabilize to the deflector limit from which it reconverges after the transient. The reasons for this are not entirely clear at present.

The simulation model has ignored measurement noise, however further simulation tests have shown that the scheme is fairly robust to Gaussian white noise. Actuator slew-rate limits and phase lags have not been included in the model. Initial testing indicates that these do affect the performance and can even cause the ESC to not converge to the minimum. This is potentially a severe limitation. Another major limitation is the assumption that the drag can be measured. This is difficult in practice, especially given that due to operational limitations, instrumentation would need to be confined to the cab since the operators usually do not own the trailer. Transmission torque measurement is often available in modern engine information systems; potentially, an observer could be constructed to estimate the drag based on this measurement combined with gradient information. The feasibility of this remains for further work.

The greatest limitation is probably the need for a the sinusoidal excitation. Although this could be turned off once the system was in steady operation, the additional energy and engineering complexity required for the excitation would need to be balanced against the potential fuel savings from the system.

\section{REFERENCES}

[1] K. R. Cooper, "A wind tunnel investigation into the fuel savings available from the aerodynamic drag reduction of trucks," DME/NAE Quarterly Bulletin, vol. 3, no. 1976, 1976.

[2] H. Gotz and G. Mayr, "Commercial vehicles," in Aerodynamics of Road Vehicles: from fluid mechanics to vehicle engineering, W.-H. Hucho, Ed. Elsevier, 1987, pp. 295-354.

[3] W. S. Saunders, "Apparatus for reducing linear and latera wind resistance in a tractor-trailer combination vehicle," Patent US3 241876A, Mar. 22, 1966. [Online]. Available: https://www.google.com/patents/US3241876

[4] F. Buckley, C. Marks, and W. Walston, "A study of aerodynamic methods for improving truck fuel economy," University of Maryland \& National Science Foundation (U.S.), College Park, MD, Tech. Rep. NSF/RA780680, 1978

[5] K. R. Cooper, "Commercial vehicle aerodynamic drag reduction: Historical perspective as a guide," in The Aerodynamics of Heavy Vehicles: Trucks, Buses, and Trains, R. McCallen, F. Browand, and J. Ross, Eds. Berlin: Springer, 2004, pp. 9-28
[6] C. J. Beers, "Air drag reducer for motor freight vehicles," Patent US4 047 747A, Sep. 13, 1977. [Online]. Available: https://www.google.com/patents/US4047747

[7] P. B. S. Lissaman and J. H. Lambie, "Air decelerator for truck cab," Patent US3 934923A, Jan. 27, 1976. [Online]. Available: https://www.google.com/patents/US3934923

[8] W. Hucho, "Introduction to automobile aerodynamics," in Aerodynamics of Road Vehicles: From Fluid Mechanics to Vehicle Engineering, W.-H Hucho, Ed. Elsevier, 1987, pp. 1-46.

[9] K. R. Cooper, "Truck aerodynamics reborn: Lessons from the past," $S A E$ Technical Paper, no. 2003-01-33, 2003.

[10] J. Barden, "Active aerodynamic control of heavy goods vehicles," $\mathrm{PhD}$ Thesis, Cranfield University, 2013.

[11] S. Windsor, "Real world drag coefficient - is it wind averaged drag?" in International Vehicle Aerodynamics Conference, Loughborough, UK, 2014, pp. 3-17.

[12] A. Seifert, O. Stalnov, D. Sperber, G. Arwatz, V. Palei, S. David, I. Dayan, and I. Fono, "Large truck drag reduction using active flow control," in The Aerodynamics of Heavy Vehicles II: Trucks, Buses, and Trains, ser. Lecture Notes in Applied and Computational Mechanics, F. Browand, R. McCallen, and J. Ross, Eds. Springer, 2009, vol. 41, pp. 115-133.

[13] L. Henning and R. King, "Drag reduction by closed-loop control of a separated flow over a bluff body with a blunt trailing edge," in 44th IEEE Conference on Decision and Control, and the European Control Conference 2005, Seville, Spain, Dec. 2005, pp. 494-499.

[14] J. F. Beaudoin, O. Cadot, J. L. Aider, and J. E. Wesfreid, "Bluff-body drag reduction by extremum-seeking control," J. Fluids Struct., vol. 22, pp. 973-978, 2006.

[15] B. K. Ariyur and M. Krstic, Real-Time Optimization by Extremum Seeking Control. Hoboken, NJ: Wiley, 2003.

[16] L. Henning, R. Becker, G. Feuerbach, R. Muminovic, R. King, A. Brunn, and W. Nitsche, "Extensions of adaptive slope-seeking for active flow control," Proc. IMechE J. Syst. Contr. Eng., vol. 222, no. 5, pp. 309-322, 2008.

[17] J. F. Beaudoin, O. Cadot, J. E. Wesfreid, and J. L. Aider, "Feedback control using extremum seeking method for drag reduction of a 3D bluff body," in IUTAM Symposium on Flow Control and MEMS, ser. IUTAM Bookseries, J. F. Morrison, D. M. Birch, and P. Lavoie, Eds. Dordrecht, The Netherlands: Springer Netherlands, Sep. 2008, vol. 7, pp. 365-372.

[18] M. Pastoor, L. Henning, B. Noak, R. King, and G. Tadmor, "Feedback shear layer control for bluff body drag reduction," J. Fluid Mech., vol. 608, pp. 161-196, 2008.

[19] N. Kamagata, S. Horio, and K. Hishida, "Drag reduction for blunt body with cross flow by extremum-seeking control," in Proc. ASME Fluids Engineering Division Summer Conference (FEDSM 2008), Jacksonville, FL, 2009, pp. 499-506.

[20] R. D. Brackston, A. Wynn, and J. F. Morrison, "Extremum seeking to control the amplitude and frequency of a pulsed jet for bluff body drag reduction," Exp. Fluids, vol. 57, no. 159, 2016.

[21] C. Zhang and R. Ordóñez, Extremum-Seeking Control and Applications: A Numerical Optimization-Based Approach. Springer, 2012.

[22] P. F. Blackman, "Extremum-seeking regulators," in An Exposition of Adaptive Control, J. H. Westcott, Ed. New York, NY: Macmillan, 1962 , pp. 36-50.

[23] M. Krstic and H. H. Wang, "Stability of extremum seeking feedback for general nonlinear dynamic systems," Automatica, vol. 36, no. 4, pp 595-601, 2000.

[24] S. J. Liu and M. Krstić, Stochastic Averaging and Stochastic Extremum Seeking. Springer, 2012.

[25] "The tyre: Rolling resistance and fuel savings," Société de technologie Michelin, 2003. [Online]. Available: https://books.google.co.uk/books?id=uv2vAAAACAAJ

[26] G. F. Franklin, J. D. Powell, and A. Emami-Naeini, Feedback Control of Dynamic Systems, 6th ed. Pearson, 2060.

[27] W. H. Moase, C. Manzie, and M. J. Brear, "Newton-Like ExtremumSeeking for the Control of Thermoacoustic Instability," IEEE Trans. Autom. Control, vol. 55, no. 9, pp. 2094-2105, 2010.

[28] G. Papageorgiou, "Adaptive flow control for minimizing the aerodynamic drag of heavy vehicles," MSc Dissertation, Cranfield University, Bedfordshire, U.K., 2016. 
2018-11-01

\section{Extremum seeking control for truck drag reduction}

\section{Papageorgiou, Georgios}

Georgios Papageorgiou, Jason Barden, James F. Whidborne and Kevin P. Garry. Extremum seeking control for truck drag reduction. Proceedings of the 2018 UKACC 12th International Conference on Control (CONTROL 2018), 5-7 September, Sheffield

https://doi.org/10.1109/CONTROL.2018.8516808

Downloaded from Cranfield Library Services E-Repository 